\title{
REVUE
}

\section{L'IDENTIFICATION DU FROMAGE DE ROQUEFORT}

\author{
par \\ G. GÉNIN, \\ Ingéniour E. P. C. I.
}

Le fromage de Roquefort est fabriqué en France depuis plusieurs siècles et même peut-être depuis Pline. On l'obtient en partant de lait de brebis et les fabriques de Roquefort se trouvent surtout situées, comme on le sait, dans le département de l'Aveyron. La région productrice occupe un plateau situé à une altitude d'environ 800 à 1.200 mètres et le climat de cette région est très variable, puisque la température moyenne en hiver ne dépasse pas 2 à $4^{\circ} 5 \mathrm{C}$., alors qu'en été elle atteint 17 à $20^{\circ} \mathrm{C}$. La quantité de pluie s'élève à la valeur moyenne de $1 \mathrm{~m}$. par an, et la principale industrie de cette région est l'élevage des moutons.

Le lait le plus apprécié pour la fabrication du roquefort est celui qui est fourni par une race originaire des Pyrénées : Ovis aries iberica. Le rendement en lait des animaux varie, suivant la nourriture et certains autres facteurs, entre 30 et 100 litres par saison, le chiffre moyen étant de 60 litres. Les brebis sont traites 2 fois par jour, et le lait est recueilli et utilisé dans des fabriques locales dispersées sur toute la région des hauts plateaux. Le fromage est préparé dans ces petites fabriques et de là, il est transporté dans les caves situées à Roquefort, où il subit une maturation.

L'agent essentiel de cette maturation est le Penicillium roqueforti, moisissure bleue verte qui se développe dans ces caves. La température est généralement réglée entre $4^{\circ} 5$ et $10^{\circ}$, l'humidité atteint $100 \%$ et une ventilation naturelle permet de changer complètement l'atmosphère des caves, au moins 3 fois par jour.

Si on se rapporte à l'étude de Marre, qui en 1906, avait fait paraître un ouvrage, sur la question du Roquefort, qui est encore resté un livre classique, le lait servant à la fabrication du fromage de Roquefort provenait à cette époque pour $97,36 \%$ de brebis, pour $2,46 \%$ de vache, et $0,18 \%$ de chèvre. Il est d'ailleurs probable que l'emploi de ce lait de vache ou de chèvre était dû à un manque de surveillance, car dès cette époque, des décrets avaient interdit formellement l'emploi d'autres laits que le lait de brebis pour la fabrication de fromage de Roquefort. Rappelons d'ailleurs que le Parlement a voté le 28 juillet 1925 une loi prescrivant l'emploi exclusif du lait de brebis pour la fabrication du fromage de Roquefort, instituant un contrôle rigoureux, de telle sorte qu'on peut 
admettre aujourd'hui que le fromage est fait pratiquement entièrement en partant de lait de brebis.

La saveur particulière du fromage de Roquefort est due au lait employé pour sa fabrication et à la maturation qu'il subit dans l'atmosphère des caves existant à Roquefort, atmosphère qu'il est pratiquement impossible de reproduire ailleurs. La saveur un peu poivrée que possède le fromage est due en outre à sa haute teneur en acide caprylique et en acide caprique.

Etant donné la réputation du fromage de Roquefort, il n'est pas étonnant que l'on ait cherché à imiter ce produit. On a done d'abord essayé de préparer en France, au Danemark, et dans d'autres pays, un fromage analogue au Roquefort, mais fabriqué en partant de lait de vache. Ce fromage est vendu sous le nom de bleu (bleu d'Auvergne) en France, en Belgique, en Orient, sous le nom de "Queso azul " en Espagne et en Amérique du Sud, etc. Le nom de bleu est également celui qui a été admis aux Etats-Unis pour définir un fromage analogue préparé en partant de lait de vache par l'Iowa State College. Le fromage bleu a un prix moins élevé que le Roquefort, car le lait de vache est beaucoup plus abondant que le lait de brebis, et comme il est difficile de faire une distinction entre les deux fromages, lorsqu'un et l'autre ne sont pas rapprochés, il s'en suit que les fraudes ont été très nombreuses.

La question s'est donc souvent posée de trouver une méthode sûre et rapide permettant de faire une distinction entre le véritable fromage de Roquefort et les produits analogues à base de lait de vache. Cette distinction pose non seulement un problème commercial, mais également un problème national et il est surtout intéressant de contrôler la vente du fromage, lorsque cette vente se fait sous la forme de parts qui ne permettent pas de contrôler les marques d'origine du produit.

II nous a donc semblé intéressant de reproduire ici une étude effectuée aux Etats-Unis par R. D. Garard, A. Mrusky, J. H. BAKER et V. PAScALE d'une part au New-Jersey College pour jeunes filles et d'autre part dans les services techniques des Gar-Baker Laboratories Inc. Ces Auteurs ont tout d'abord pensé que les différences qui existent entre les matières grasses du lait de vache et du lait de brebis doivent se retrouver dans le fromage. A cet effet, ils ont passé en revue différents travaux qui ont été faits sur ces produits et nous indiquerons dans le tableau I comment il est possible de faire une première distinction entre le lait de vache et le lait de brebis, d'après leur teneur en acide caprylique et en acide caprique. 


\section{TABLEAU I.}

Acide capryli- Acide capi-

Origine du lait que concentra- rique concention molécu-
laire en $\%$
culaire en $\%$ lé-

Vache $\ldots \ldots \ldots \ldots \ldots$.

$\begin{array}{rrr}2,0 & 3,0 & \text { Dhingra } \\ 5,8 & 10,1 & \text { Dhingra }\end{array}$

Brebis ................

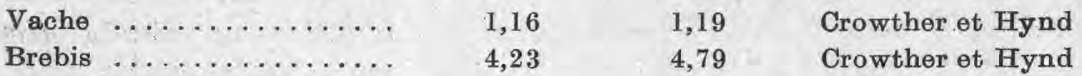

Ces valeurs ne sont pas-en parfait accord, mais elles montrent que le lait de brebis contient une beaucoup plus haute teneur en acides caprylique et caprique.

Or l'indice de Polenske correspond essentiellement à la teneur en acides caprylique et caprique. Il était donc également intéressant de déterminer cet indice sur un certain nombre de produits dérivés du lait de vache ou du lait de brebis. Les indications concernant l'indice de Polenske des produits provenant de lait de vache sont nombreuses; par contre elles sont beaucoup plus rares en ce qui concerne le lait de brebis. On a réuni toutefois dans le tableau II ci-dessous les valeurs qu'on a pu trouver dans la littérature.

\section{TABLEAU II.}

\section{Produit}

Matière grasse extraite du beurre .....

Matière grasse extraite du beurre......

Matière grasse extraite du beurre .....

Beurre australien.............

Beurre danois

Beurrégyptien

Beurre de vache

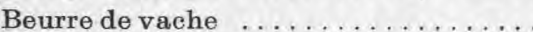

Beurre devache................

Fromage de Roquefort ..........

Matière grasse extraite du lait de brebis

Roquefort :

Elite $\ldots \ldots \ldots \ldots \ldots \ldots \ldots$

Belier ..................

Mialane et Cie .............

Louis Rigal ...............

Fromage de Roquefort ..........

Fromage de lait de brebis .........

Lait de brebis .............

Matière grasse du lait . . . . . . . . .
Lait de vache

Lait de brebis

$$
\begin{aligned}
& 1,35 / 3,00 \\
& 1,50 / 3,00 \\
& 2,0 \\
& 1,4 / 2,3 \\
& 2,1 \\
& 1,9 / 2,7 \\
& 1,7 / 3,1 \\
& 1,46 / 2,43 \\
& 1,90 / 2,60
\end{aligned}
$$

$4,9 / 6,5$

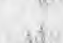

Les chiffres de ce tableau montrent qu'en général, la matière grasse provenant du lait de brebis a un indice de Polenske nettement 
supérieur à la matière grasse provenant du lait de vache. Les chiffres de la dernière ligne du tableau montrent toutefois qu'on a enre. gistré parfois un indice de Polenske de 5,8 pour la matière grasse provenant du lait de vache et un indice de Polenske de 2,2 pour la matière grasse extraite du lait de brebis, mais il est difficile d'établir quelle valeur il faut attacher à ces dernières déterminations. La méthode décrite par PoLEnske pour la détermination de l'indice est en effet tout à fait empirique. Des modifications nombreuses ont été apportées au mode opératoire et il est normal que les déter. minations faites par des expérimentateurs n'employant pas la même méthode donnent parfois des résultats un peu contradictoires.

Les indications qui figurent dans le tableau II montrent également que si la matière grasse a subi le phénomène de maturation, son indice de Polenske reste néanmoins plus élevé dans le cas de la matière grasse du lait de brebis.

Dans le but d'apprécier dans quelle limite on peut établir des déductions précises d'après la valeur de l'indice de Polenske, GARARD et ses collaborateurs ont étudié un grand nombre de produits laitiers provenant du lait de brebis et du lait de vache, et ils ont déterminé, en adoptant un procédé rigoureusement standard, l'indice de Polenske de ces différents produits.

L'Association des chimistes agricoles experts des Etatis-Unis a publié, il y a quelques années, deux méthodes pour l'extraction de la matière grasse des produits laitiers, en vue de l'examen de constantes de cette matière grasse. L'une de ces méthodes consiste à traiter le produit avec une solution à $5 \%$ de potasse, l'autre à le traiter arec une solution à $50 \%$ d'acidesulfurique. Dans les deux cas, la caséine est dissoute et il suffit de séparer la matière grasse par une opération mécanique.

La méthode alcaline s'est révélée inapplicable dans le cas qui nous intéresse, celui de l'examen des fromages, car ces produits renferment des acides gras libres qui auraient été perdus par le dosage, par suite de leur neutralisation par la potasse. On a done fait appel à la méthode de traitement par les acides, mais comme l'acide sulfurique concentré peut décomposer la matière grasse, il a été fait appel à une technique moins brutale que celle décrite par les chimistes de l'Association américaine.

Le fromage, après avoir été maintenu à $25^{\circ}$ pendant quelques heures, est malaxé avec les doigts et tamisé. On applique alors le fromage, au moyen d'une spatule, sur les parois latérales d'un grand bécher, de façon à laisser le fond de ce bécher complètement libre. On place le bécher dans une étuve à $45 / 50^{\circ}$, et on l'y laisse toute une nuit. La matière grasse se sépare sous la for- 
me d'une couche limpide et, dans certains cas, on constate avec le fromage fraîchement préparé qu'il se dépose également au fond du récipient une couche de petit-lait. On recueille la matière grasse que l'on sépare du petit-lait par centrifugation, et on peut alors effectuer sur le produit ainsi isolé la détermination des indices caractéristiques.

Avec certains échantillons de roquefort, il avait été impossible d'appliquer cette méthode et il avait fallu procéder à l'extraction de la matière grasse au moyen de l'éther.

Une fois cette technique opératoire pour la séparation de la matière grasse parfaitement établie, les Auteurs dont nous résumons les travaux, ont tout d'abord vérifié que les différences qui existent entre les indices de Polenske du lait de brebis et du lait de vàche n'étaient pas dues à la nourriture souvent différente que reçoivent ces animaux. A cet effet, des vaches et des brebis ont reçu pendant plusieurs mois consécutifs la même nourriture et on a déterminé sur le lait fourni par ces animaux l'indice de Polenske des matières grasses. Les résultats qui figurent dans le tableau III ci-dessous montrent que ces différences ne peuvent être dues à la nourriture et qu'elles sont bien le résultat d'une différence fondamentale entre les constitutions des matières grasses du lait de vache et du lait de brebis.

\section{TABLEAU III.}

INDIGE DE POLENSKE DE LAITS FOURNIS PAR DES ANIMAUX REGEVANT LA MEME NOURRITURE.

$\begin{array}{llc}\text { Matière grasse provenant de } & \text { Vache } & \text { Brebis } \\ \text { Lait } \ldots \ldots \ldots \ldots \ldots \ldots \ldots \ldots & 2,15 & 4,5 \\ \text { Beurre } \ldots \ldots \ldots \ldots \ldots \ldots \ldots & 2,25 & 4,6 \\ \text { Lait } \ldots \ldots \ldots \ldots \ldots \ldots \ldots & 1,95 & 4,85 \\ \text { Lait } \ldots \ldots \ldots \ldots \ldots \ldots \ldots & 1,5 & 3,7 \\ \text { Beurre } \ldots \ldots \ldots \ldots \ldots \ldots \ldots & 1,45 & 4,7 \\ \text { Beurre } \ldots \ldots \ldots \ldots \ldots \ldots \ldots & 1,9 & 3,7\end{array}$

On a ensuite vérifié si ces différences existaient également entre les matières grasses extraites du fromage et ayant par conséquent subi une maturation. Nous nous contenterons de reproduire quelques chiffres déterminés sur des fromages français qui montrent qu'indiscutablement, il existe une différence très nette entre les produits provenant de lait de vache et ceux fabriqués en partant de lait de brebis. 
TABLEAU IV.

\section{NDICE DE POLENSKE DE DIFFÉRENTS FROMAGES.}

Marque de fromage Indice de Polenske

Coloration de la

I - Fromages de lait de vache.

matière grasse

Bleu français :

\begin{tabular}{|c|c|c|}
\hline A. L. 1 N. . . . . . & 1,45 & jaune \\
\hline A. L. 2 N........ & 1,55 & jaune \\
\hline Aulait ........... & 1,65 & jaune \\
\hline Bison ............. & 2,6 & jaune \\
\hline Casino ............. & 2,1 & jaune \\
\hline Peyrelade (1935) . . . . & 1,6 & jaune \\
\hline Peyrelade (1935).... . & 1,75 & jaune \\
\hline Peyrelade (1936)..... & 2,3 & jaune \\
\hline Riom (1935) ....... & 1,6 & jaune \\
\hline $\operatorname{Riom}(1936) \ldots \ldots \ldots$ & 1,8 & jaune \\
\hline Riom (1936) ....... & 1,75 & jaune \\
\hline . & 2,8 & jaune \\
\hline nembert & 1,8 & jaune \\
\hline
\end{tabular}

Roquefort :

II - Fromages de lait de brebis

\begin{tabular}{|c|c|}
\hline Coudère & 5,1 \\
\hline Coudère & 5,5 \\
\hline Jeanne d'Arc ........ & 5,35 \\
\hline Le Bélier . . . . . . . . & 4,15 \\
\hline Le Bélier . . . . . . . . . & 5,5 \\
\hline Le Bélier . . . . . . . . . & 5,1 \\
\hline Le Phare .......... & 5,3 \\
\hline Puma ........... & 5,2 \\
\hline Puma ........... & 3,7 \\
\hline Puma ........... & 3,6 \\
\hline Société Dee ........ & 5,0 \\
\hline Société Mondiale .... & 4,5 \\
\hline Société Nouvelle . . & 5,95 \\
\hline Star (Rigal) . . . . . . & 4,1 \\
\hline
\end{tabular}

vert pâle
vert pâle
vert pâle
vert pâle
vert pâle
vert pâle
vert pâle
vert pâle
vert pâle
vert pâle
vert pâle
vert pâle
vert pâle
vert pâle

Il résulte de ces chiffres que l'hypothèse d'après laquelle la matière grasse d'un fromage fabriqué en partant de lait de vache a un indice de Polenske dont la valeur maximum ne dépasse jamais 3, semble justifiée. La matière grasse, pour ce dosage, peut être séparée soit par fusion, comme nous l'avons décrit, soit par extraction au moyen d'un acide. Des déterminations analogues ont été faites en partant de produits d'origines diverses et dans tous les cas, cette règle a été confirmée, ce qui semble démontrer que la présence d'acides caprylique et caprique dans le lait de brebis est indépendante du pays dans lequel vivent les animaux, de la nourriture qu'ils reçoivent et du procédé de maturation du fromage. 
A cette valeur de l'indice de Polenske, s'ajoute une autre caractéristique qui est due à ce qu'une matière grasse ayant un faible indice de Polenske est toujours jaune dorée, tandis qu'un produit ayant un indice de Polenske supérieur à 3 présente fréquemment un reflet verdâtre. La différence de coloration entre la matière grasse fournie par une vache et la matière grasse fournie par une brebis a d'ailleurs été déjà signalée par PALMER qui a trouvé que les vaches concentrent des caroténoïdes dans leur lait, alors que chez les brebis, ce phénomène n'a pas lieu.

Enfin, pour compléter cette investigation, GARARD a également étendu ses recherches à des fromages préparés pendant des années différentes et, dans tous les cas, il a constaté que le Roquefort donne une matière grasse dont l'indice de Polenske est toujours compris entre 3,6 et 5,95 .

Il semble done possible de dire avec certitude que tout fromage à moisissure bleue, qui possède un indice de Polenske inférieur à 3 , n'est pas du fromage de Roquefort. Une confirmation de cette conclusion pourra être établie en considérant également la coloration de la matière grasse.

Les spécialistes semblent donc désormais en possession d'un moyen rapide permettant de différencier les fromages de lait de brebis des fromages de lait de vache, mais le procédé que nous venons de signaler ne permet pas d'établir d'une façon précise si un fromage a été préparé en partant d'un mélange de lait de vache et de lait de brebis et dans quelle proportion ces différents laits ont été mélangés; nous croyons savoir que des études sont actuellement en cours, afin de résoudre ce problème.

\section{BIBLIOGRAPIIIE ANALYTIQUE}

\section{LES LIVRES}

Babonneix (L.). - Les régimes chez l'enfant. I volume broché, 604 pages, tableaux. Edité chez Masson et Cie, 120, boulevard Saint-Germain, Paris, 1936. Prix : 75 francs.

Nous sommes très heureux de présenter ce nouveau et très important ouvrage du $\mathrm{D}^{\mathrm{P}} \mathrm{BABONNEIX}$, ouvrage constituant une véritable mise au point. Si ce travail s'adresse plus particulièrement au corps médical et spécialement au pédiâtre, il intéresse encore tous ceux que préoccupe la question de l'hygiène du lait. Il est, par ailleurs, utile que les producteurs et les industriels laitiers connaissent l'opinion de l'éminent spécialiste qu'est l'auteur du livre : "L'es régimes chez l'enfant ".

Le premier chapitre est d'ordre biologique. Il a paru, en effet, indispen- 